\title{
EGLISE ET ETAT \\ Cadre juridique et enjeux actuels de la relation entre l'Eglise catholique et la République Démocratique du Congo
}

\author{
Par Kitengie Kasongo François ${ }^{1}$
}

\section{INTRODUCTION}

"Les Eglises ne peuvent guère, écrit DE NAUROIS, faire l'économie de droit étatique, il faut à leurs biens une assiette juridique, leurs activités intéressent l'ordre public dont l'Etat porte la responsabilité $»^{2}$

La République Démocratique du Congo, notre pays, tourne péniblement les pages les plus sombres de son histoire, tissées au fil de temps par la convergence des multiples facteurs endogènes et exogènes. En effet, des héritages historiques lourds, très lourds, à partir des crises répétées des légitimités et maintient des pouvoirs, des échecs des développements, des guerres atroces et répétées qui l'ont achevé en retardant sa maturation de plusieurs décennies en sont la preuve la plus éloquente.

En fait, ce constat amer que fait tout congolais conscient de la situation de notre pays, incite à un devoir de savoir, de réflexion, de pouvoir et d'agir pour arrêter ce processus dangereux d'effondrement continuel de notre société congolaise. Dès lors, il serait donc mal indiqué, pour les fils et filles de ce pays, de baisser les bras et de courber l'échine devant cette menace de liquéfaction de toute une nation, aujourd'hui objet de railleries, des jalousies et des convoitises de toutes parts.

A cet égard, l'Eglise catholique qui fonde son enseignement sur l'amour du prochain, milite à côté d'autres acteurs de la vie sociale, pour l'instauration d'un Etat de droit où règnent la paix et la justice.

Solidement implantée dans toutes les provinces du pays depuis l'époque coloniale, l'Eglise catholique de la République Démocratique du Congo, à travers ses nombreux mouvements et associations, a répondu aux besoins de la population en matière d'enseignement, de santé et de développement. C'est autant dire que, l'engagement de l'Eglise catholique en faveur du bien être de la population dans notre pays ne date pas d'aujourd'hui; il remonte à l'époque coloniale où la collaboration entre l'Eglise et l'Etat était un fait indéniable au nom de la mission civilisatrice. En effet, l'on sait que, l'importation ou l'implantation de l'Etat en Afrique centrale était fondée sur l'impératif de la « mission civilisatrice » où, civiliser,

1 Assistant à la Faculté de Droit de l'Université de Lubumbashi.

2 De NAUROIS, L, «Eglise et Etat » in Encyclopaedia universalis, Corpus7, Paris, UE France1990, p. 976. 
EGLISE ET ETAT Cadre juridique et enjeux actuels de la relation entre l'Eglise catholique et la République Démo-

signifiait diffuser la civilisation chrétienne occidentale. Dans ce contexte, il appartenait donc à l'Etat de déterminer la qualité de compatibilité où non compatibilité des cultes à l'impératif de la «mission civilisatrice».

Cependant, à partir de l'indépendance, et surtout avec le régime MOBUTU et la proclamation de la laïcité, l'Etat se démarqua des anciennes alliances coloniales dans une parfaite dissociation des intérêts de l'Etat et des Eglises, méconnaissant ainsi unilatéralement l'ensemble des accords et traités intervenus entre le Congo belge et le Saint-Siège, les conventions de droit public interne, les missions religieuses et des traités coloniaux, le texte législatif et réglementaire ainsi que, le texte constitutionnel qui régissait les rapports entre l'Eglise catholique et l'Etat.

Pourtant, il est de principe que les conventions légalement formées tiennent lieu des lois à ceux qui les ont faites ${ }^{3}$ et que, en cas de succession d'Etats, l'Etat successeur hérite les traités et accords internationaux légalement ratifiés par l'Etat prédécesseur. ${ }^{4}$

Cela étant, la préoccupation majeure de la présente réflexion est de circonscrire le cadre juridique et l'enjeu actuel de la relation entre l'Eglise catholique et la République Démocratique du Congo, dans le but d'amener les deux institutions à collaborer efficacement pour l'avènement d'un Etat de droit dans notre pays.

Pour ce faire, il va falloir jeter un regard rétrospectif sur l'histoire de la relation entre l'Eglise catholique et l'Etat pendant la période coloniale et post - coloniale.

\section{UN BREF APERÇU DE L'EGLISE CATHOLIQUE DE LA REPUBLIQUE DEMOCRATIQUE DU CONGO}

La configuration globale de l'Eglise catholique du Congo étant largement tributaire de la colonisation belge qui a marqué le pays ${ }^{5}$, il nous semble qu'on ne peut mieux circonscrire le cadre juridique et l'enjeu actuel de la relation entre les deux institutions, sans s'intéresser au cadre historique de la République Démocratique du Congo, et celui de leur relation avec ladite Eglise, pendant et après l'indépendance.

1.1. Le cadre historique de la République Démocratique du Congo avant l'indépendance.

Pourquoi allait-on coloniser? Pour quels calculs?

En réponse à ces deux questions, Bruno MUPINGANAYI KADIAKUIDI répond en ces termes : « c'est pour le motif matériel et le motif spirituel qui ont agi intimement entre-

3 Article 33 du code civil congolais livre III.

4 Jean Pacifique BALAAMO MOKELWA, Eglises et Etat en République Démocratique du Congo. Fondements juridiques et jurisprudence (1876-2006), éd. L'Harmattan, Paris, 2009, p. 209.

5 WAMU OYANTAMBWE, Eglise catholique et pouvoir politique au Congo Zaïre, éd. L'Harmattan, Paris, 1997, p.15. 
lacés $»^{6}$. En effet, il y a eu d'abord, surenchérit-il, le motif matériel et le motif spirituel et en un mot, c'est pour la religion qu'on a souvent, dans l'ancien temps colonisé.?

A cet effet, dans un texte bien connu, écrit le même auteur, que la bulle du pape Alexandre VI, datée de l'an 1493, il est marqué déjà expressément et solennellement que les portugais et les espagnols pourront conquérir les pays lointains mais à condition de les catéchiser, et à la condition de les moderniser, de propager la foi et en propageant les mœurs. ${ }^{8}$

Dès lors, l'on comprend pourquoi, au Congo, après la fondation de l'Etat, surenchéritil, Léopold II fit appel à tous les ordres religieux de Belgique ${ }^{9}$. En effet, l'autorité morale des missionnaires était assez grande pour transformer la mentalité des indigènes en ce qui concerne l'hygiène, la prévoyance et le monde métaphysique. Ils contribuèrent plus que quiconque au relèvement intégral de la race noire par un contact journalier avec les habitants dont ils parlaient les langues, par la connaissance qu'ils avaient de leurs besoins et par les services qu'ils leurs rendaient avec dévouement, tant sous le rapport religieux et moral que matériel. Dans cette optique, les missions constituaient les pôles de développement et un socle sur lequel toute l'œuvre de la colonisation reposait. En contre partie, les missionnaires reçurent des autorités politiques, la mission d'instruire sur toute l'étendue de la colonie, les blancs et les noirs avec les subventions et sous le contrôle du gouvernement.

Par ailleurs, étant donné la position stratégique des missionnaires dans l'œuvre de la colonisation, il était exclu que le gouvernement prenne une quelconque importante décision concernant la colonie, sans avoir consulté les représentants missionnaires qui occupaient des sièges importants du congrès colonial, organe de conception et de décision de mise en valeur du Congo belge; leur autorité morale sur les indigènes et leur rapprochement avec ces derniers constituaient une arme d'inflexion des voix des gouvernants coloniaux ${ }^{10}$.

Cela est d'autant vrai qu'aux dires de WANU OYATAMBWE, Léopold II convaincu de l'importance de l'action des missionnaires catholiques décida, lors de la cession de l'Etat Indépendant du Congo à la Belgique en 1908, de fonder son action colonisatrice sur trois piliers, dont le premier était l'Eglise catholique, dite Eglise nationale, chargée de pacifier les cœurs des indigènes en les convertissant au christianisme; le deuxième fut, le pilier commercial et l'exploitation des richesses du pays, et le troisième était l'administration et son armée. ${ }^{11}$

Comme on peut le constater, la collaboration entre l'Eglise catholique et l'Etat colonial était quasi parfaite, à telle enseigne qu'aux dires de WANU OYATAMBWE, l'Eglise catho-

6 Bruno MUPINGANAYI KADIAKUIDI, Réhabilitation de l'Eglise catholique dans sa mission apostolique face au foisonnement des églises et des guerres d'agression en RDC, éd. CARI, Kinshasa, 2008, p.

7 Ibidem.

8 Ibidem.

9 Ibidem.

10 Bruno MUPINGANAYI KADIAKUIDI, $\boldsymbol{O} \boldsymbol{p}$. Cit, p.45.

11 WAMU OYANTAMBWE, $\boldsymbol{O} \boldsymbol{p}$. $\boldsymbol{C} \boldsymbol{i t}, \mathrm{p} .45$. 
EGLISE ET ETAT Cadre juridique et enjeux actuels de la relation entre l'Eglise catholique et la République Démo-

lique dans l'affaire du « caoutchouc rouge » préféra se murer dans un silence prudent pour sauvegarder son rôle de premier pilier de l'aventure coloniale, contrairement aux missionnaires protestants d'origine Britannique et Américaine qui avaient dénoncé cette situation avec la première énergie. ${ }^{12}$

En fait, il faut noter que, la collaboration entre l'Eglise catholique et l'Administration coloniale n'était pas dépourvu d'un cadre juridique bilatéral expresse. En effet, des nombreux textes juridiques régissant les rapports entre les deux institutions, au nom de la mission civilisatrice, ont été signés aussi bien par le saint siège et la Belgique, que par cette dernière et les autorités de l'Eglise sur le plan interne ${ }^{13}$.

En tout état de cause, il ya lieu de noter que pendant la période d'avant l'indépendance, la collaboration entre l'Eglise catholique et l'Etat avait un cadre juridique bien précis, attesté par des nombreux textes juridiques dont ceux que nous avons énumérés à titre exemplatif. Cette relation, faut-il le dire, avait comme enjeu majeur, la mission civilisatrice que l'administration coloniale ne pouvait accomplir sans le concours de l'Eglise catholique. Par ailleurs, cette dernière ne pouvait non plus réussir dans son entreprise, sans le concours de l'Etat ni à l'abri du contrôle de ce dernier.

Au fait, il va sans dire que, les relations entre l'Eglise catholique et l'Etat à cette étape de l'histoire du Congo, vont suivre le principe « do et des » qui résume les enjeux de leur étroite collaboration. ${ }^{14}$

\section{LE CADRE JURIDIQUE DE LA RELATION ENTRE L'EGLISE CATHOLIQUE ET LA REPUBLIQUE DEMOCRATIQUE DU CONGO}

Quelle est la législation qui fonde et justifie l'action de l'Eglise dans l'histoire du peuple Congolais?

Répondre à cette épineuse question ou chercher à situer le cadre juridique de la relation entre l'Eglise Catholique et l'Etat en République Démocratique du Congo, nécessite une longue réflexion autour des différents accords et traités ainsi que les textes législatifs et réglementaires qui ont existé depuis l'époque coloniale jusqu'à nos jours.

12 Ibidem, p. 15-16.

13 A ce propos, Jean Pacifique BALAAMO MOKELWA, Op. Cit., p. 109, rapporte les textes suivants : la convention entre la Belgique et le saint siège au sujet du Congo belge de 1953 adaptant celle de 1906 entre l'EIC et le saint siège aux réalités nouvelles du pays d'une part, et d'autre part, cherchant à uniformiser les références juridiques et résoudre la question de la diversité de la législation spéciale; ensuite, le décret de 1888 qui définit les institutions religieuses comme des institutions publiques religieuses créées par l'Etat en partenariat avec les sociétés missionnaires.

14 WAMU OYANTAMBWE, $\boldsymbol{O} \boldsymbol{p} . \boldsymbol{C} \boldsymbol{i t}$, p.16. 


\subsection{LES GRANDES ORIENTATIONS DE LA LEGISLATION CONGOLAISE SUR LES ACTIVITES ET INSTITUTIONS RELIGIEUSES}

L'on ne peut prétendre résoudre la problématique du cadre juridique qui fonde l'intervention de l'Eglise Catholique au Congo dans le domaine politique sans prendre en considération tout l'arsenal juridique du droit congolais de religion ainsi que l'ensemble de textes y afférant depuis l'Etat indépendant du Congo jusqu'à nos jours.

En effet, il s'avère évident que le régime léopoldien a jeté les premières assises du droit congolais et en a donné l'orientation. Ici, les accords et traités permettaient de vivre ensemble pour les forces en présence sur le nouvel espace étatique. Les accords et traités constituent le gros de textes législatifs de l'Etat Indépendant du Congo (E.I.C.).

Sur ce, notons avec Jean PACIFIQUE BALAAMO MOKELWA que plus de quatrevingts dix pourcent de dispositions législatives du régime régalien était constitué de diverses conventions ${ }^{15}$.

Le régime Léopoldien de cultes et missions est un régime essentiellement conventionnel. Le fil conducteur de la politique religieuse de l'E.I.C. ressort clairement à travers toutes les conventions :

1. La fusion du temporel et du spirituel, du civil et du religieux, de l'Etat et des missions religieuses dans la campagne de la mission de civilisation.

2. la subordination des missions religieuses à l'Etat.

3. la préférence nationale.

4. la liberté religieuse est garantie pour encourager les communautés religieuses intéressantes à s'établir au Congo.

Toutefois, il convient de signaler que, ces conventions obligent en vertu des principes du droit international de « pacta sunt servanda » et de la « succession des Etats ». Elles constituent, pour ainsi dire, le gros de la législation spéciale qui a traversé toute l'histoire de la République Démocratique du Congo grâce au principe de continuité juridique en droit Congolais.

A cet effet, le décret sur les organisations sans but lucratif de 1888 constitue une référence historique, non seulement parce qu'il pose le jalon du droit des associations en droit congolais mais surtout, parce qu'il demeure jusqu'à nos jours une référence à laquelle renvoient les dispositions législatives, mais aussi parce qu'elles évoquent des institutions ou des associations qui ne sont pas du droit commun ou des législations spéciales. ${ }^{16}$

La convention entre la Belgique et le Saint Siège au sujet du Congo Belge de 1953 voulait adapter celle de 1906 entre l'E.I.C. et le Saint Siège aux nouvelles réalités du pays d'une part, et d'autre part elle cherchait à uniformiser les références juridiques et résoudre la question de la diversité de la législation spéciale. Elle devrait créer un cadre légal unique

15 Jean PACIFIQUE BALAAMO MOKELWA, Op.Cit, 208-209.

16 Cfr décret loi sur les organisations sans but lucratif de 1888. 
EGLISE ET ETAT Cadre juridique et enjeux actuels de la relation entre l'Eglise catholique et la République Démo-

à tous les missions et cultes reconnus. Cette convention n'a jamais été ratifiée par le Parlement Belge ${ }^{17}$.

La convention de gestion des écoles nationales du 26 février 1977 est un arrangement entre les leaders religieux et les autorités civiles sur la question scolaire après l'imbroglio créé par la mise en application brutale de l'idéologie révolutionnaire de recours à l'authenticité. La question dépassait de loin le problème de gestion des écoles publiques; elle consistait plutôt à créer un cadre scolaire propice à l'idéologie révolutionnaire de désaliénation mentale de la jeunesse par la voie de l'enseignement public.

C'est ainsi que pour ne pas hypothéquer l'avenir du pays par la faillite de son système éducatif, des négociations furent entreprises pour la rétrocession des écoles publiques. ${ }^{18}$

Par ailleurs, notons que le droit conventionnel demeure un droit sectoriel qui n'a jamais créé un cadre global pour une collaboration et une cohabitation harmonieuses entre les églises et le pouvoir public dans divers domaines.

A côté des accords et traités, il faut relever l'omniprésence de l'esprit du décret de 1888 dans toutes les lois postérieures sur les associations. Les institutions de ce décret, souligne Jean Pacifique BALAAMO MOKELWA n'ont jusqu'ici trouvé un autre cadre légal; cet oubli a fini par créer un vide juridique qui est préjudiciable pour le pays ${ }^{19}$. Les institutions dans l'esprit du décret de 1888 sont les activités et les institutions créées par les pouvoirs publics ou dont la gestion peut être confiée aux associations religieuses, philanthropiques et scientifiques. Les institutions religieuses sont des institutions publiques religieuses créées par l'Etat en partenariat avec les sociétés missionnaires.

Cependant, comme il faut le constater, c'est depuis le $19^{\text {ème }}$ siècle que l'évangélisation agressive du Congo avait été dominée par des sociétés missionnaires protestantes qui ne sont liées à aucune entité centrale comme dans l'Eglise catholique où il existe un pouvoir central représenté par le Saint siège qui coordonne l'ensemble des activités missionnaires. Le fait de ne pas être rattaché à une église a, sans aucun doute, poussé les sociétés missionnaires protestantes à être beaucoup plus entreprenantes malgré le protectionnisme de la politique religieuse coloniale de préférence nationale Belge.

L'Etat Indépendant du Congo (E.I.C.) était une cible privilégiée des sociétés missionnaires protestantes de tout bord. Les missions religieuses catholiques étant des circonscriptions ecclésiastiques catholiques créées par l'Etat en partenariat avec le Saint Siège et confiées à la gestion des Sociétés Missionnaires catholiques, appelées en termes propres ordres et instituts missionnaires, qui en ont la compétence juridique selon le droit canonique.

Soulignons qu'en réalité, le décret de 1888 était un cadre légal pour tous les établissements d'utilité publique dénommés alors « Organismes sans but lucratif ». Ces institutions étaient des établissements d'utilité publique créés par des législations spéciales et ne pou-

17 Cfr. La convention entre la Belgique et le saint siège de 1953.

18 Cfr. La convention sur la gestion des écoles nationales du 26 février 1977

19 Jean Pacifique BALAAMO MOKELWA, Op. Cit, p.209. 
vant pas être mises de ce fait dans le même sac que les associations. Le décret sur les associations de 1959 mit un terme à la confusion des « organismes sans but lucratif pour créer un cadre légal commun aux associations sans but lucratif ». Les troubles liés à la décolonisation n'ont pas permis à la loi sur les associations de 1959 de produire tous les effets escomptés et elle fut vite dépassée.

La loi sur les associations de 1965 est une adaptation du décret de 1959 à la nouvelle situation juridique créée par l'accession du pays à la souveraineté internationale.

En outre, la loi de 2001 sur les associations sans but lucratif et les établissements d'utilité publique porte sur les associations et établissements nés de l'initiative privée. Alors que l'association sans but lucratif naît d'une volonté collective, l'établissement d'utilité publique peut naître de la volonté d'une seule personne.

Il faut noter que, la loi de 2001 met ensemble la loi de 1965 et les lois sur l'exercice des cultes de 1971 et de 1979 pour donner un cadre légal aux associations confessionnelles.

Les établissements d'utilité publique de la loi de 2001 sont d'une autre nature juridique que les institutions du décret de 1888. Le vide juridique créé par la loi des associations est toujours manifeste.

Les nombreux textes législatifs et réglementaires se sont accumulés tout au long de l'histoire de la République Démocratique du Congo, et portent sur les avantages accordés aux institutions et associations religieuses sous forme de subsides ou de subventions publiques, d'exemptions fiscales ou d'exonérations, qui constituent un financement public direct ou indirect de l'Etat aux associations pour les œuvres d'intérêts publics qu'elles accomplissent.

Durant la période postcoloniale, le manque d'un Etat fort jouissant de toutes les prérogatives régaliennes n'a pas permis au législateur de statuer sur la question de financement des activités et institutions religieuses.

Le renvoi des nouveaux textes législatifs aux vieux décrets coloniaux et régaliens dénote un certain archaïsme du droit congolais des religions et la peur d'innovation due au manque d'études doctrinales et jurisprudentielles qui pourraient éclairer le législateur Congolais en la matière.

Tous les textes législatifs et réglementaires revêtent un encrage de la théorie de la domination étatique, où l'Etat est jaloux de sa souveraineté et tient coûte que coûte à la gestion de l'espace public pour mieux filtrer l'offre religieuse.

Sans être ouvert, l'espace public colonial restait ouvert aux missions et cultes reconnus au nom du titre de civilisation. Alors que la loi ne reconnaissait pas des associations de fait, pour raison d'ordre public, la puissance coloniale prit une réglementation sévère contre des associations de fait d'indigènes. Plusieurs furent dissoutes et interdites car la puissance coloniale les prenait pour des mouvements subversifs (secte ou association indigène hiérarchisée, secte prophétique hiérarchisée). Elles furent dissoutes et interdites parce qu'elles étaient contraires à la civilisation et constituaient une menace à la tranquillité et à l'ordre public. Durant la période postcoloniale, l'ordre public fut souvent évoqué comme motif pour la police des activités religieuses. 
EGLISE ET ETAT Cadre juridique et enjeux actuels de la relation entre l'Eglise catholique et la République Démocratique du Congo

2.1.1. La police des activités et institutions religieuses

a) La police coloniale des activités et institutions religieuses

A ce niveau, signalons que l'article 76 du Code Pénal de 1888 est la première disposition générale qui garantit la protection de la liberté des cultes. Cet article dispose : « seront punis d'une servitude pénale de huit jours à deux ans et d'une amande de vingt cinq à cinq cents francs ou d'une de ces peines seulement toutes personnes qui, par des violences, outrages ou menaces, par des troubles ou des désordres, auront porté atteinte à la liberté des cultes ou à leur libre exercice, et à la liberté de conscience $»^{20}$

Ce texte sera repris in extenso par le Code Pénal de 1940 en son article 179. Dans la fièvre de la décolonisation, une ordonnance - loi fut promulguée le 25 mars 1960 contre les actes d'intolérance raciale et religieuse qu'on enregistrait un peu partout dans la colonie. En son article $1^{\mathrm{er}}, 1^{\prime}$ ordonnance - loi n ${ }^{\circ} 25-131$, dispose :

«Les gestes, les paroles ou les écrits susceptibles de provoquer, d'entretenir ou d'aggraver la tension entre races, ethnies ou confessions sont punis d'une peine de servitude pénale d'un mois à un an et d'une amende n'excédant pas 3.000 Francs ou d'une de ces peines seulement. $"^{21}$

b) La police postcoloniale des activités et institutions religieuses

La police postcoloniale des activités et institutions religieuses fut fondée sur une disposition du code pénal du 30 janvier 1940 qui porte sur les atteintes à la liberté des cultes en son article 179 .

Il existe de nombreuses dispositions législatives ou réglementaires qui déterminent les infractions contre l'ordre public. L'article $1^{\text {er }}$ de l'ordonnance - loi $n^{\circ} 72-039$ du 30 août 1972 dispose :

"la section $x$ du titre IV du Code Pénal Livre II, intitulée de quelques autres infractions contre l'ordre public, est complétée par un article 155 quater ainsi libellé; sera puni d'une servitude pénale de six mois à cinq ans, tout officiant qui, lors du baptême d'un adepte zaïrois, lui conférera une appellation aux consonances étrangères. $»^{22}$

Abordant dans le même sens, Clément MAKIOBO faisant allusion au mémorandum de l'épiscopat du Zaïre qui avait été rejeté par le Président Mobutu note que, "le Bureau Politique avait aussi confirmé la décision antérieure relative aux noms ». Tous les congolais (Zaïrois) qui sont nés à partir du 16 février seront baptisés avec des noms authentiquement

20 PIRON, P. et DEVOS, J., Codes et lois du Congo Belge, Bruxelles, Léopoldville. Maison Ferdinand Larcier, éditions des Codes et lois du Congo Belge, 1954, p. 297.

21 Les Codes Larciers République Démocratique du Congo Tome II, Droit Pénal, Bruxelles, Larcier Afrique, Editions 2003, p.38.

22 J.O., 5, 1973, p. 329. 
zaïrois. Tout prêtre qui se permettrait d'imposer aux enfants Zaïrois des noms d'emprunt, s'exposerait à des poursuites judiciaires conformément aux stipulations prévues par l'ordonnance - loi en cette matière. $»^{23}$

Signalons à cet effet que le 5 septembre 1974, le M.P.R. est proclamé comme une «Eglise » et son fondateur, Mobutu comme »le messie » du peuple Zaïrois. A partir de cette date, l'Etat s'en prend particulièrement à l'église catholique en lui enlevant non seulement ses moyens de communication sociale et ses écoles mais aussi en détruisant des objets d'art chrétiens sur les lieux publics. C'était donc tout le fondement de la culture catholique qui était visé par la politique de Mobutu ${ }^{24}$.

Au regard de l'article 27 de la Constitution de 1967, note Jean - Pacifique BALAAMO MUKELWA, le Président pouvait exercer le pouvoir exécutif par voie d'ordonnance ${ }^{25}$. Au moment fort de la dictature de Mobutu, l'application de cette disposition constitutionnelle fut parfois exagérée et accompagnée des violations graves portant atteinte aux libertés publiques. Une série de mesures furent prises et exécutées, les unes furent coulées sous forme de loi et les autres ne l'ont jamais été ${ }^{26}$ :

- La nationalisation des universités et des instituts supérieurs par l'ordonnance - loi n ${ }^{\circ} 71 / 075$ du 6 août $1971^{27}$.

- L'obligation d'abandonner les noms à consonance européenne formulée sous la loi $\mathrm{n}$ ${ }^{\circ} 73 / 022$ du 20 juillet 1973 relative au nom de personnes physiques ${ }^{28}$.

- La dissolution de l'association sans but lucratif dénommée «Comité Permanent des Evêques du Zaïre » par l'ordonnance $n^{\circ} 73-021$ du 15 février $1973^{29}$.

- La suppression des jours fériés légaux religieux et leur remplacement par des nouvelles commémorant des événements importants de la révolution zaïroise authentique initiée par le Président Mobutu ${ }^{30}$.

TSHIZANGA MUTSHIPANGU observe à ce sujet que :

"Les convictions religieuses lorsqu'elles quittent le domaine du for intérieur pour se projeter sur la scène publique doivent être conciliées avec d'autres considérations et

23 Clément MAKIOBO, op. cit., p.238.

24 Ibidem, p. 192.

25 Jean - Pacifique BALAAMO MOKELWA, op. cit., p.166.

26 Ibidem, p.166.

27 Cfr M.C., 20, 1971, pp.929-936.

28 Cfr J.O., 22, 1975, p.1405- 1406.

29 Cfr Loi nº87-010 du $1^{\text {er }}$ août 1987 portant Code de la famille, in J.O., numéro spécial, 1987, p. 49.

30 Cfr « Ordonnance n $71-326$ du 31 décembre 1971 fixant les jours fériés légaux » in J.O., 2, 1972, p. 54 - 55, « Ordonnance n ${ }^{\circ} 72 / 63$ du 14 septembre 1972 fixant les jours fériés légaux », in J.O., 22, 1972, pp. $700-701$; « Ordonnance $n^{\circ} 73 / 340$ du 26 octobre 1973 complétant l'ordonnance $n$ ${ }^{\circ} 72 / 363$ du 14 septembre 1972 fixant les jours fériés légaux » in J.O., 1, 1974, p.3. 
EGLISE ET ETAT Cadre juridique et enjeux actuels de la relation entre l'Eglise catholique et la République Démocratique du Congo

la liberté de conscience cesse alors d'être un concept absolu pour devenir une liberté susceptible de limitation et de restriction ${ }^{31} »$.

L'ordre public est défini, note GHESTIN JACQUES comme : « le bon fonctionnement des instructions indispensables à la collectivité » ${ }^{32} \mathrm{Il}$ s'analyse également surenchérit-il, en termes « d'ensemble des valeurs estimées indispensables par un pays pour son fonctionnement général et le bien commun de la population. $»^{33}$

a) Le droit à la liberté religieuse dans les constitutions congolaises

Il est vrai que plusieurs textes constitutionnels ont régi à des époques différentes notre pays, ont consacré la liberté religieuse. La loi fondamentale du 17 juin 1960 en son article 12 dispose :

" toute personne a droit à la liberté de pensée, de conscience et de religion, à la liberté de changer de manifester sa religion ou sa conviction ou sa conviction individuellement ou collectivement, en public ou en privé, par le culte, la propagation, l'enseignement, les pratiques et l'accomplissement des rites. $»^{34}$

2. Aucun élève d'un établissement d'enseignement ne sera astreint à suivre des cours d'instruction religieuse, à participer à une cérémonie religieuse ou à prendre part à un culte procédant d'une religion autre que sienne ${ }^{35}$.

3. Les dispositions ci-dessus ne font pas obstacle à l'application des mesures prévues par la loi ou les édits pour répondre aux nécessités imposées dans une société démocratique par la sécurité publique, protection de l'ordre, de la santé ou de la morale publique ou de celle des droits et libertés d'autrui. »

Dans le même ordre d'idées, l'article 24 de la Constitution de 1964 proclame : «Toute personne a droit à la liberté de penser, de conscience et de religion. Dans la République, il n'y a pas de religion d'Etat. Toute personne devenue majeure a le droit de manifester sa religion ou ses convictions, seule ou en commun, tant en privé qu'en public, par le culte, l'enseignement, les pratiques, l'accomplissement des rites et l'Etat de vie religieuse, sous réserve du respect de l'ordre public et de bonnes mours. $»^{36}$

31 TSHIZANGA MUTSHIPANGU, Libertés religieuses dans l'ordonnancement juridique congolais, in les analyses juridiques, 2006, $\mathrm{N}^{\circ}$ 8, pp. 27-39.

32 GHESTIN JACQUES, Traités de droit civil, les obligations. Les contrats L.G.D.J., Paris, 1980, n 93, p. 66.

33 TSHIZANGA MUTSHIPANGU, op. cit., p. 38.

34 Article 12 de la loi fondamentale du 17 juin 1960.

35 Ibidem.

36 Article 26 de la Constitution de Transition de Sun City de 2003 et l'article 17 de l'Acte Constitutionnel de Transition de 1994. 
La même disposition fut reprise par la Constitution de 1967. L'article 10 de cette Constitution de 1967 est une reprise du texte de l'article 24 de la Constitution de $1964^{37}$. L'article 17 de l'Acte constitutionnel de transition de 1994 est une reformulation de la disposition constitutionnelle de 1964, en son article 17 et la constitution de transition de Sun City de 2003, en son article 26, vont en restructurer la formulation : «Dans la république, il n'y a pas de religion d'Etat. »

Toute personne a le droit de manifester sa religion selon ses convictions, seule ou en commun, tant en public qu'en privé, par le culte, l'enseignement, les pratiques, l'accomplissement des rites et l'état de vie religieuse, sous réserve de l'ordre public et des bonnes mœurs. La loi fixe les conditions de constitution des associations religieuses.

Signalons ici que la Constitution de transition de Sun City de 2003 à son article 26 est une reprise du texte de l'article 17 de l'acte constitutionnel de transition de $1994^{38}$.

A la différence des textes constitutionnels qui l'ont précédé le décret - loi constitutionnel nº03 du 27 mai 1997 relatif à l'organisation et à l'exercice du pouvoir en république Démocratique du Congo était muet sur la liberté religieuse. Son article 2 signale TSHIZANGA MUTSHIPANGU disposait que « En République Démocratique du Congo, l'exercice des droits et libertés individuels et collectifs est garanti sous réserve du respect de la loi, de l'ordre public et de bonnes mœurs. $»^{39}$

Disons donc que sa formulation autorisait à admettre qu'il englobait la liberté religieuse. La loi $n^{\circ} 004 / 2001$ du 20 juillet 2001 portant dispositions générales applicables aux associations sans but lucratif et aux établissements d'utilité publique est plus explicite à ce sujet lorsque elle indique à l'article 46 que : «toute personne a le droit de manifester sa religion ou ses convictions, l'accomplissement des rites et l'état de vie religieuse sous réserve de l'ordre public et de bonnes mœurs "».

Cette disposition est identique au prescrit de l'article 26 alinéa 2 de la constitution du 4 avril 2003 qui énonce que « toute personne a droit à la liberté de penser, de conscience et de religion, toute personne a le droit de manifester sa religion ou ses convictions, seule ou en commun, tant en public qu'en privé, par le culte, l'enseignement, les pratiques, l'accomplissement des rites et l'état de vie religieuse, sous réserve de l'ordre public et de bonnes mœurs.

Enfin l'article 22 de la constitution de 2006, en vigueur est presque une reproduction du même texte de l'article 26 de la constitution de transition de Sun City 2003. « Dans la république il n'y a pas de religion d'Etat. Toute personne a le droit de manifester sa religion ou ses convictions, seul ou en commun, tant en public qu'en privé, par le culte, l'enseignement, les pratiques, l'accomplissement des rites et l'état de vie religieuse sous réserve de

37 TSHIZANGA MUTSHIPANGU, op. cit., p. 30.

38 Journal officiel, $\mathrm{n}^{\circ}$ spécial du 5 avril 2003.

39 Article 22 de la Constitution de 2006. 
EGLISE ET ETAT Cadre juridique et enjeux actuels de la relation entre l'Eglise catholique et la République Démocratique du Congo

l'ordre public et de bonne mœurs ». La loi fixe les conditions de constitution des associations religieuses ${ }^{40}$.

b) Le droit à la liberté religieuse au niveau international

Bien que n'ayant pas une portée juridique positive, note Jean Pacifique BALAAMO MUKELWA, la déclaration universelle des droits de l'homme du 10 décembre 1948 avait fait l'objet d'une recommandation à l'Assemblée Générale des Nations Unies ${ }^{41}$. A cet effet, la charte des Nations Unies approuvée par la République Démocratique du Congo par sa publication dans le Journal Officiel d'avril 1999.

L'article 18 de la Déclaration Universelle de Droits de l'Homme stipule : « toute personne a droit à la liberté de changer de religion ou de convention ainsi que la liberté de manifester sa religion ou sa conviction, seule ou en commun, tant en public qu'en privé, par l'enseignement, les pratiques, le culte et l'accomplissement des rites ${ }^{42}$ ».

Adopté le 16 décembre 1966 par l'Assemblée Générale des Nations Unies et entré en vigueur le 23 mars 1976, le pacte international relatif aux droits civils et politiques à son article 2 dispose :

« Les Etats parties au présent pacte s'engagent à respeter et à garantir à tous les individus se trouvant sur leur trritoire et relevant de leur compétence les droits reconnus dans le présent acte, sans distinction aucune, notamment de race, de couleur, de sexe, de langue, de religion, d'opinion politique ou de toute autre option, d'origine nationale ou sociale, de fortune, de naissance ou de toute autre situation ${ }^{43}$ ».

Le 26 juin 1981 à Nairobi au Kenya, la Charte africaine des droits de l'homme et des

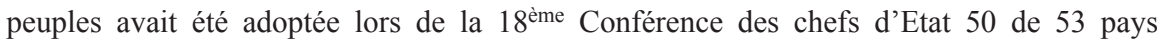
membres de l'Organisation de l'Unité Africaine 'OUA) parmi lesquels la République Démocratique du Congo. La dite charte africaine à son article 8 dit :

«La liberté de conscience, la profession et pratique de la religion, sont garanties. Sous réserve de l'ordre public, nul ne peut être l'objet de mesures de contrainte visant à restreindre la manifestation de ces libertés. " ${ }^{44}$

Le texte constitutif de l'Union Africaine qui est devenu officiellement opérationnel le 26 mai 2001, réaffirmée solennellement son engagement à l'égard de la charte africaine de droits de l'homme et des peuples parmi les objectifs que s'est fixé l'Union en son articlez 3, il est dit

40 TSHIZANGA MUTSHIPANGU, op. Cit, p. 30.

41 Article 18 de la Déclaration Universelle de Droit de l'Homme.

42 Article du texte constitutif de l'Union africaine, 1981.

43 Article 3 du texte constitutif de l'Union africaine, 1981.

44 Jean-Pacifique BALAAMO MOKELWA, op. cit., p.121. . 
" promouvoir et protéger les droits de l'homme et des peuples conformément à la charte africaine des droits de l'homme et des peuples d'autres instruments pertinents relatifs aux droits de l'homme. " ${ }^{45}$

Nous ne pouvons pas clôturer ce point sans dire un mot sur la laïcité de l'Etat Congolais.

\subsection{LA LAÏCITE DE L'ETAT CONGOLAIS}

Notons d'entrée de jeu que, la laïcité de l'Etat se traduit par le caractère non confessionnel de 1 'Etat ${ }^{46}$. Cela veut dire que l'Etat n'a pas de religion. Il est neutre vis-à-vis de toutes les religions.

Certes, le principe constitutionnel de laïcité fut consacré pour la première fois d'une manière explicite par la constitution révisée du 15 août 1974 en son article $1^{\text {er }}$ : «La République du Zaïre est un Etat unitaire, démocratique, social et laïque ${ }^{47}$ »

Depuis le principe de laïcité sera observé dans le constitutionnalisme Congolais. La constitution de transition révisée de Sun City de 2003 reprit la formule déjà utilisée par l'acte constitutionnel de la transition de 1994 qui dispose : «La République Démocratique du Congo est dans ses frontières du 30 juin 1960, un Etat indépendant, souverain, indivisible, démocratique, social et laïc ».

Pour sa part, la Constitution de 2006 en son article $1^{\text {er }}$ dispos : «La République Démocratique du Congo, et dans ses frontières du 30 juin 1960, un Etat de droit, indépendant, souverain, uni et indivisible, social, démocratique et laïc $»^{48}$

Notons avec TSHIZANGA M. que la laïcité de l'Etat oblige celui-ci à ne pas s'immiscer dans l'exercice de la liberté religieuse ou de discriminer les religions

Bien plus, surenchérit-il, elle interdit aux personnes fréquentant les institutions publiques d'aborder les signes extérieurs ostentatoires ou traduisant un prosélytisme.

Au demeurant, la neutralité de l'Etat, qui est négative, n'empêche pas celui-ci de réglementer la création des associations culturelles, confessionnelles ou religieuses.

A ce propos, TSHIZANGA M. souligne que, la référence à Dieu qui est contenue dans le serment que doit prêter le chef de l'Etat pose le problème de la laïcité de l'Etat. Cela s'explique par les prescrits de l'article 74 .

L'article 74 alinéa 2 du projet de Constitution de la République Démocratique du Congo énonce que « avant son entrée en fonction, le président de la République prête, devant la Cour Constitutionnelle, le serment ci-après : Moi ... Elu Président de la République Démocratique du Congo, je jure solennellement devant Dieu et la notion... »

45 Article $1^{\text {er }}$ de la Constitution de 2006.

46 TSHIZANGA MUTSHIPANGU, op. Cit, p. 33.

47 Ibidem, p. 3.

48 Article $1^{\text {er }}$ de la Constitution de 2006. 
EGLISE ET ETAT Cadre juridique et enjeux actuels de la relation entre l'Eglise catholique et la République Démo-

Dans le même sens le préambule dudit projet de constitution évoque Dieu lorsqu'il énonce que « nous, peuple congolais ... conscient de nos responsabilités devant Dieu, la nation, l'Afrique et le monde. »

Eu égard à ce qui précède, notons que le recours aux différentes références juridiques sur lesquelles se sont fondées les relations Eglise et Etat dans l'histoire tumultueuse de la République Démocratique du Congo, cela nous a permis de préciser que, le droit Congolais des religions a produit une abondante littérature juridique fortement complexe dans l'histoire. Le foisonnement de genre dans la littérature juridique couvre plusieurs domaines du droit international, droit privé, droit public, droit constitutionnel, droit pénal, droit administratif, droit social, droit fiscal, etc.

\section{SITUATION ACTUELLE DE LA RELATION ENTRE L'EGLISE CATHOLIQUE ET LA REPUBLIQUE DEMOCRATIQUE DU CONGO}

Au regard des analyses précédentes, il convient de noter que l'histoire de la relation entre l'Eglise catholique et la République Démocratique du Congo est non seulement complexe, mais aussi difficile et mouvante. ${ }^{49}$

En effet, sous la colonisation, l'Etat collaborait étroitement sur la base d'un cadre juridique précis, en vue de la réalisation de la mission civilisatrice. Chacune des parties agissaient dans les limites de la ligne tracée par l'arsenal juridique régissant leur tâche respectives, lequel comprenait les traités et accords avec le saint siège, les textes bilatéraux ainsi que les actes réglementaires et autres textes signés entre l'Etat et la hiérarchie ecclésiale congolaise.

Cependant, après l'indépendance, l'évolution de leur relation a été différente, pour ne pas dire multiraciales. En effet, les prises de position du régime MOBUTU avec comme corollaires le renforcement de la dictature, l'étatisation des institutions jadis dévolues à l'Eglise, ainsi que la politique de l'authenticité et la détérioration du tissu socio -politique et économique du pays, ont davantage creusé un fossé entre les deux institutions au détriment du développement intégral de la nation congolaise et, partant du bien être de sa population.

$\mathrm{Au}$ jour d'aujourd'hui, il est intéressant de noter que, la relation entre l'Eglise catholique et l'Etat congolais reste encore tributaire de la situation post-coloniale. Ces deux institutions ne sont pas dans une harmonie susceptible de favoriser une orientation commune dans le sens de la réalisation des objectifs de justice, de paix et d'amour.

En effet, le manque d'un cadre juridique clairement exprimé, depuis la proclamation de la laïcité qui a jeté dans les oubliettes tous les accords et traités sus-évoqués n'a pu que contribuer au maintien de la suspicion, de la méfiance, et donc, d'une action parallèle entre les deux institutions, dont la collaboration serait pourtant nécessaire pour inscrire notre pays dans un régime démocratique, respectueux de la dignité et de la liberté de tous et de chacun. 
C'est autant dire que, l'Eglise catholique en République Démocratique du Congo, n'agit actuellement qu'en vertu des prérogatives reconnues aux autres composantes de la société civile, car avec la laïcité, il n'y a plus la religion d'Etat. Pourtant, comme l'a si bien souligné Jean Pacifique MOKELWA BALAAMO, les conventions sur lesquelles se sont fondées les alliances au sein de l'appareil de domination coloniale constituent le cadre juridique de référence protégé par le droit international. En effet, l'EIC et le saint siège, deux sujets de droit international, ont formaté ces références en une convention bilatérale. ${ }^{50}$

A cet égard, estimons-nous, il y a un manque à gagner, si l'Etat congolais revenait dans l'histoire de sa collaboration avec ladite Eglise, en vue d'y puiser de l'abondante richesse résultant de l'expertise, du dynamisme et de la détermination des structures de celle-ci.

Cela étant, il en va, à notre humble avis, de l'intérêt du peuple congolais, de voir la République Démocratique du Congo, leur pays, s'inscrire dans la dynamique de la promotion de Etat de justice, de paix et d'amour.

Ce qui ne peut être possible que si, ses dirigeants reprenaient le chemin et l'orientation de l'Eglise qui ne cesse d'insister, à travers ses multiples messages, sur l'avènement d'un Etat de Droit.

A cet effet, estimons-nous, il serait souhaitable que, les dirigeants de l'Etat congolais renégocient un cadre juridique nouveau avec cette Eglise, en tenant compte des réalités actuelles et en vue de mettre en place des structures de coordination de leur action commune, dans le but de réaliser l'enjeux majeur, qui est celui de l'avènement d'un Etat où règne la justice, la paix et l'amour longtemps souhaité, tant par l'Eglise catholique que par la nation congolaise toute entière.

A cet égard, l'Eglise par ses structures, à savoir la conférence épiscopale du Congo et ses commissions, notamment Caritas -développement, justice et paix offre une aubaine à l'Etat Congolais de trouver un point de départ et un terrain d'action favorable à la réalisation des objectifs de développement intégral de la population congolaise.

\section{CONCLUSION}

La présente réflexion s'est focalisée autour du cadre juridique et de l'enjeu actuel de la relation entre l'Eglise catholique et la République Démocratique du Congo.

Elle a eu comme prétention de circonscrire un cadre de collaboration harmonieuse entre les deux institutions, dont l'action concertée a été d'une importance capitale dans l'instauration de l'Etat moderne dans cette partie de l'Afrique centrale.

Pour ce faire, nous avons, d'entrée de jeu, démontré que, la relation entre les deux institutions dont l'une est spirituelle et, l'autre temporelle, a connu des variations multiformes.

A l'époque coloniale, les deux institutions ont eu à collaborer harmonieusement sur la base d'un cadre juridique, constitué des traités et accords bilatéraux intervenus entre leSaint-Siège; d'une part, et Léopold II, la Belgique ou le Congo belge d'autre part et, c'est 
EGLISE ET ETAT Cadre juridique et enjeux actuels de la relation entre l'Eglise catholique et la République Démo-

dans le but d'instaurer un Etat moderne avec comme enjeu majeur : apporter la civilisation aux populations indigènes, vivant sur le territoire de ce qu'on a jadis appelé, Etat Indépendant du Congo.

Cependant, avec l'accession de la République Démocratique du Congo à la souveraineté internationale et, surtout le renforcement du régime dictatorial du Maréchal MOBUTU qui a largement contribué à la détérioration du tissu sociopolitique et économique de ce pays, ainsi que la proclamation de la laïcité, les deux institutions ont eu à entretenir des relations tantôt tumultueuses, tantôt harmonieuses. En effet, l'Etat Congolais, fort et jaloux de sa laïcité et de sa souveraineté, a jeté dans les oubliettes tout l'arsenal juridique qui a régulé leur apport avec l'Eglise catholique et faciliter ainsi son implantation au centre de l'Afrique. Depuis lors, l'Eglise catholique, consciente de son rôle et de sa position incontestable dans ce pays, n'a pu, pour sa part, agir autrement que de prendre une orientation dénonciatrice des abus de la classe politique qu'elle considère comme des obstacles à l'avènement d'un Etat de droit en République Démocratique du Congo.

Devant cet état de choses, nous nous sommes posé une série de questions, notamment de quel droit l'Eglise catholique peut-elle prendre une telle orientation face à un état laïc? Quelle pourrait être l'orientation utile à la réalisation des objectifs de paix, de justice et, donc de respect de la dignité humaine au sein de ce pays, dont le défis à relever sur les plans développemental et autres, s'affichent de plus en plus nombreux?

En réponse à ces questions, nous avons démontré que, par respect des principes de «Pacta sunt servenda » et de la " succession d'Etats aux traités », la République Démocratique du Congo, ne devrait pas unilatéralement jeter purement et simplement dans les oubliettes les traités et accords sus-évoqués, ni ignorer le rôle joué par l'Eglise catholique pendant la période coloniale.

En revanche, l'Eglise catholique, consciente de sa mission de pacifier les cœurs et de promouvoir l'instauration d'une société fondée sur les valeurs respectueuses de la dignité humaine, avons-nous souligné, devrait ne pas se cantonner dans une position dénonciatrice sans impact sur la situation concrète de la population.

Aussi, avons-nous estimé, en dépit des difficultés qui ont pollué la relation entre les deux institutions, et étant donné l'histoire de leur collaboration harmonieuse, l'Eglise catholique et l'Etat congolais, devraient tenir compte d'un fait historique évident dans l'évolution des institutions humaines : la thèse, l'antithèse et la synthèse.

Ainsi, si la période coloniale était celle de la thèse, la période poste coloniale qui a culminé dans l'avènement du régime MOBUTU avec tous ses corolaires, devrait être considérée comme celle de l'antithèse dans leur relation, et celle qui a suivi la chute de MOBU$\mathrm{TU}$, comme celle de la synthèse.

Dès lors, les deux institutions devraient revenir à l'histoire de leur action concertée pour y puiser ce qu'il y a de meilleur en vue d'opérer utilement en faveur de l'intérêt supérieur, à savoir le bien-être intégral de la population congolaise.

A cet effet, à l'instar de Léopold II et des autorités du Congo belge, l'Etat congolais devrait activer sa machine diplomatique dans le but de renégocier les accords et traités in- 
tervenus entre elle et la hiérarchie de ladite Eglise en vue de leur réadaptation à la situation actuelle. Une telle démarche, avons-nous estimé, loin d'être en contradiction avec l'objectif de l'Etat laïc en République Démocratique du Congo, constituerait un manque à gagner car, l'on ne peut s'en douter, l'Eglise catholique, en dépit de toute absence d'un cadre juridique opérationnel devant régir actuellement leur relation avec l'Etat congolais, reste une institution importante dans ce pays et un acteur incontournable dans la mission de la réalisation du développement de tout homme et de tout l'homme, pourtant dévolu à la République Démocratique du Congo.

C'est pourquoi, Léon Bloy l'avait qualifié en son temps comme la torche en ces termes : « Nous voici donc, la torche à la main, pour une tentative d'embrassement universel. Il n'y a peut-être jamais d'occasions meilleures, une plus favorable époque. A l'échéance de dix - neuf siècles, on a fini par découvrir que le catholicisme est, enfin de compte, ce qu'il y a de moins absurde, les expériences de la prétendue liberté religieuse dans les quatre derniers cents ans n'ayant abouti qu'à l'idiotification et à l'avilissement de la race humaine. Quelques-uns mêmes, dont le nombre grandit chaque jour, sentent déjà que des récentes et trop fameuses conversions littéraires ou sentimentales ne suffisent pas, que c'est le catholicisme intégral, absolu, sans accommodement ni retour possible, qui est exigé maintenant, et que c'est bien décidemment le feu qui est nécessaire à une société menacée de putréfaction. $»^{51}$

\section{BIBLIOGRAPHIE}

I. Les accords et traités

a) La Déclaration Universelle de Droit de l'Homme

b) L'acte constitutif de l'Union africaine 1981

c) La convention entre la Belgique et le saint siège de 1953

d) La convention sur la gestion des écoles nationales du 26 février 1977

II. Instruments juridiques

Les textes législatifs et réglementaires

a) La constitution révisée du 15 aout 1974

b) La constitution de 1974

c) L'acte constitutionnel de transition de 1994

d) La constitution de 1967

e) La constitution de transition de Sun City de 2003

f) La Constitution de 2006

51 Léon BLOY, Le Mendient ingrat. Mon journal, quatre ans de captivité à cochons-sur marne. L'invendable, éd. Robert Lafon, Paris, 1999, p. 540. 
EGLISE ET ETAT Cadre juridique et enjeux actuels de la relation entre l'Eglise catholique et la République Démo-

g) La loi fondamentale du 17 juin 1960

e) Les textes législatifs

a) Les Codes Larciers de la République Démocratique du Congo Tome II, Droit Pénal, Bruxelles, Larcier - Afrique

b) PIRON, P. et DEVOS, J., Codes et lois du Congo Belge, Bruxelles, Léopoldville. Maison Ferdinand Larcier, éditions desCodes et lois du Congo Belge

c) Le code civil congolais livre III

d) Loi n87 - 010 du 1er août 1987 portant Code de la famille, in J.O., numéro spécial, 1987

e) L'Ordonnance n71-326 du 31 décembre 1971 fixant les jours fériés légaux

f) L'Ordonnance $n^{\circ} 72 / 63$ du 14 septembre 1972 fixant les jours fériés légaux

g) L'Ordonnance $n^{\circ} 73 / 340$ du 26 octobre 1973 complétant l'ordonnance $n^{\circ} 72 / 363 \mathrm{du}$ 14 septembre 1972 fixant les jours fériés légaux

h) Le décret loi sur les organisations sans but lucratif de 1888

\section{OUVRAGES}

a) De NAUROIS, L, «Eglise et Etat » in_Encyclopaedia universalis, Corpus7, Paris, UE France 1990

b) Léon BLOY, Le Mendient ingrat. Mon journal, quatre ans de captivité à cochons-sur marne. L'invendable, éd. Robert Lafon, Paris, 1999

c) GHESTIN JACQUES, Traités de droit civil, les obligations. Les contrats L.G.D.J., Paris, 1980

d) WAMU OYANTAMBWE, Eglise catholique et pouvoir politique au Congo Zaïre, éd. L'Harmattan, Paris, 1997

e) Bruno MUPINGANAYI KADIAKUIDI, Réhabilitation de l'Eglise catholique dans sa mission apostolique face au foisonnement des églises et des guerres d'agression en

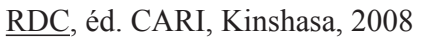

f) Jean Pacifique BALAAMO MOKELWA, Eglises et Etat en République Démocratique du Congo. Fondements juridiques et jurisprudence (1876-2006), éd. L'Harmattan, Paris, 2009, p. 209.

Article

a) TSHIZANGA MUTSHIPANGU, Libertés religieuses dans l'ordonnancement juridique congolais, in les analyses juridiques, 2006 